\title{
Participación de los colaboradores holguineros en la formación de recursos humanos durante las misiones médicas internacionalistas
}

\section{Participation of collaborators from Holguín province in the formation of human resources during the international medical missions}

\author{
Pedro A. Díaz Rojas,' Magalys Moreno Montañez" \\ ' Doctor en Medicina. Máster en Educación Médica. Profesor Titular. Escuela Nacional \\ de Salud Pública. La Habana, Cuba. \\ "Doctor en Medicina. Máster en Educación Médica. Asistente. Universidad de Ciencias \\ Médicas de Holguín. Holguín, Cuba.
}

\begin{abstract}
RESUMEN
Partiendo de las ideas estratégicas definidas por Fidel en relación con la formación y preparación continua del capital humano en salud, y el desarrollo vertiginoso de la colaboración médica cubana, se realizó este trabajo de recuento histórico sobre la participación de los colaboradores de la provincia Holguín en estos procesos y en especial en la Misión Médica Cubana Barrio Adentro en la República Bolivariana de Venezuela. Se abordó la formación académica del posgrado con sus principales figuras: la especialización, maestrías y doctorado y las bases que sustentaron el desarrollo del pregrado. La participación de los colaboradores holguineros materializa la formación de capital humano en el sector de la salud, y mantiene vigente el principio de solidaridad de la revolución cubana y sus trabajadores en la meta de lograr la excelencia en los servicios de salud y cooperar activamente en la formación de los recursos humanos para Cuba y el mundo.
\end{abstract}

Palabras clave: Formación de recursos humanos en salud, educación de posgrado, colaboración, Venezuela. 


\begin{abstract}
Taking as a basis the strategic ideas defined by Fidel about the formation and permanent preparation of the human capital in the field of healthcare, and the accelerated development of the Cuban medical cooperation, a historical account was made on the participation of medical collaborators from Holguin province in these tasks, particularly Cuban medical mission known as "Barrio Adentro" in the Bolivarian Republic of Venezuela. This account addressed the post-graduate academic formation such as specialization, master's courses and PhD's courses and the foundations behind the development of the undergraduate education. The participation of collaborators from Holguin province supports the formation of human resources in the health care sector and shows the validity of the solidarity principle of the Cuban revolution and its workers.
\end{abstract}

Key words: Formation of human resources in health care, postgraduate education, cooperation, Venezuela.

\title{
SÍ NTESIS DE LAS I DEAS DE FIDEL SOBRE LOS RECURSOS HUMANOS DE LA SALUD
}

Durante los 52 años de Revolución, el pensamiento y accionar de Fidel Castro Ruz sobre la salud pública cubana siempre han estado vinculados a la garantía de la salud para todo el pueblo, la accesibilidad a todos los servicios de salud con calidad, la formación y desarrollo de su capital humano, así como a la cooperación y ayuda solidaria internacionalista. ${ }^{1}$

Durante todos estos años se ha garantizado la formación del personal de la salud requeridos para brindar una atención de calidad al pueblo cubano y crear una reserva de personal para la colaboración en salud. En tal sentido se pone de manifiesto las ideas estratégicas planteadas por Fidel en relación con la formación y desarrollo del capital humano en salud, las que se pudieran sintetizar en:

Yo quiero establecer un principio: [...] la docencia existe para la asistencia.... allí donde hay un profesor, allí donde hay un hombre obligado al estudio constante y sistemático, hay mejor asistencia. Esto es muy importante. ${ }^{2}$ (J unio 1982).

Por eso se exige [...] una gran preparación de nuestros profesores, una gran preparación de nuestros médicos. La combinación de la docencia, la investigación, la asistencia [...] Esto significa también un gran esfuerzo de superación por parte de los propios profesores [...] Porque cuando hablamos de superación de los miles de profesores de ciencias médicas, estamos hablando también de la superación de nuestra asistencia médica... Y nosotros debemos crear los mecanismos para alcanzar estos objetivos. ${ }^{2}$ (Abril 1983).

Estos propósitos resumen la necesidad de materializar sus ideas sobre el concepto de capital humano, donde expresó:

Capital humano implica no sólo conocimientos, sino también -y muy esencialmenteconciencia, ética, solidaridad, sentimientos verdaderamente humanos, espíritu de 
sacrificio, heroísmo, y la capacidad de hacer mucho con muy poco. [...] Estoy pensando en calidad, jen calidad! La vamos adquiriendo cada vez más. [...] Crear capital humano que no se agota... sabrán mucho más y se habrán multiplicado cuando reciban sus títulos, se habrán multiplicado otra vez cuando dominen una especialidad, se habrán multiplicado cuando hayan cumplido una, dos o las misiones internacionalistas que sean necesarias; se habrán multiplicado cuando tengan una maestría o un doctorado, como en un futuro no lejano lo tendrán ya masivamente nuestros médicos. ${ }^{3}$ (febrero 2006).

\section{LOS ANTECEDENTES DE LA COLABORACI ÓN MÉDICA}

La colaboración internacionalista es un principio de la revolución socialista cubana. Es el pago de la deuda a la solidaridad de los pueblos del mundo. Es la materialización de la solidaridad en su más profundo concepto.

La colaboración médica cubana data desde 1963, el año en que comienza con carácter organizado, con el envío de la primera Brigada Médica Cubana a la Republica de Argelia. No hay antecedentes de este tipo de colaboración antes de 1959. No obstante, ya en fecha temprana como el año 1960, Cuba ofrecía su primera ayuda médica internacional al enviar una brigada médica a Chile, después del terremoto que azotó a ese país.

A fines de 1962, en el acto inaugural de la Facultad de Ciencias Básicas y Preclínicas "Victoria de Girón", el Comandante en Jefe anunció al pueblo de Cuba la decisión del gobierno revolucionario de brindar ayuda médica internacional en el campo de la salud.

Entre las funciones llevadas a cabo por la colaboración médica cubana se destacan: la asistencia médica clinicoquirúrgica a la población de los países en los lugares más apartados e intrincados, el desarrollo conjunto de las campañas de educación sanitaria y de vacunación masiva de la población, el control higiénico y epidemiológico, la formación de recursos humanos en el campo de las ciencias de la salud y la prestación de servicios en brigadas médicas a damnificados por huracanes, sismos y otras contingencias en diferentes países.

Hoy Cuba brinda colaboración médica en 74 países, distribuidos en África, America Eurasia y Medio Oriente. ${ }^{4}$

\section{La Colaboración Médica en América Latina}

La mayor expresión de solidaridad e internacionalismo de la colaboración médica de Cuba surgió a finales de 1998 tras el azote del huracán Mitch a varios países de Centroamérica, principalmente a las naciones de Honduras y Guatemala.

Ante la gravedad de la situación en estos países el gobierno cubano comenzó a desarrollar el Programa Integral de Salud (PIS) para Centroamérica y el Caribe, que había sido azotado poco antes por otros fenómenos meteorológicos,se amplia posteriormente a algunos países de África y Asia. EI PIS surge como una modalidad de la colaboración médica cubana en 1998 para extender los servicios médicos gratuitos a un centenar de países, fundamentalmente de África y América Latina. 
EI PIS también incluye la formación y capacitación de recursos humanos tanto en las áreas donde laboran los galenos cubanos como aquí en Cuba. De ahí surge la Escuela Latinoamericana de Medicina.

\section{Las Facultades de Ciencias Médicas en África. I nicio de la docencia en el exterior}

En la década del 80 , se inicia una etapa de colaboración académica, con la creación de diversas facultades de ciencias médicas en el exterior. En su mayoría en los países del continente africano. Profesores de diversas especialidades, tanto de ciencias básicas biomédicas como de ciencias clínicas participaron activamente en las facultades de Angola, Guinea Bissau, Mozambique, Sudáfrica, Gambia, Eritrea y Etiopia, por mencionar algunas de ellas.

En 1979 se desarrolla el internado en Nicaragua, una experiencia importante para los cubanos, el pregrado en el exterior. Los resultados fueron muy satisfactorios, y los colaboradores holguineros participan de lleno en la organización, dirección y trabajo docente con los internos cubanos en ese país.

\section{La Colaboración en la República Bolivariana de Venezuela}

El 15 y 16 de diciembre de 1999 ocurre el deslave de Vargas, considerado el peor desastre natural ocurrido en Venezuela y Suramérica durante el siglo xx en el cual 215 mil personas fueron afectadas y 55 mil fueron desplazadas. Barrios enteros quedaron sepultados por lodo y escombros, ante este fenómeno natural Cuba decide enviar el primer grupo de médicos en función de ayuda humanitaria, con un excelente desempeño ante el cumplimiento de su deber internacionalista, aspecto decisivo para la futura misión.

La experiencia y el desempeño de los profesionales y técnicos cubanos hizo posible la solicitud del gobierno de este hermano país de continuar prestando los servicios médicos a parte de la población excluida. Se inicia con los primeros 54 especialistas en MGI, con dos años o más de experiencia en el cumplimiento de misiones en África, Centroamérica, Sudamérica y el Caribe, que se ubicaron en 10 de las parroquias más pobres de Caracas, entre ellas Catia, San Agustín, La Pastora y Sucre.

Solo traían el humanismo y la solidaridad de un pueblo que a lo largo de más de cuatro décadas ha compartido con los pobres del mundo el talento y el sacrificio de sus hijos.

En el año 2002 se extiende la experiencia a los estados de Lara, Miranda, Trujillo, Carabobo, Barinas y Guárico.

La Misión Barrio Adentro se inició el 16 de abril de 2003, fecha coincidente en el contexto internacional con el 25 aniversario de la Declaración de Alma-Atá, firmada por la Organización Mundial de la Salud en 1978 cuyo objetivo estratégico fue: "Salud para todos en el año 2000"(Historia de la Misión Médica Cubana en la República Bolivariana de Venezuela. Documento inédito. Misión Médica Cubana. 2009).

Entre los meses de abril y junio arriban a Venezuela el primer grupo de la incipiente Misión Barrio Adentro, entre julio y agosto del propio año se amplía la atención a todos los municipios de Miranda y para diciembre ya habían arribado el grueso de las brigadas médicas extendiéndose a todo el país. En el Programa Aló Presidente de ese mes el Presidente Hugo Chávez Frías oficializa públicamente la Misión Barrio Adentro. 
Paralelamente se desarrolla de forma vertiginosa la Misión Milagros, brindando atención especializada a pacientes con problemas oftalmológicos. En su seno se desarrollan las ópticas populares para dar cobertura a las necesidades de la población.

El 12 de junio de 2005, se inicia una fase superior de la Misión Barrio Adentro, con la construcción de una red de unidades integradas por los Centros Médicos de Diagnóstico Integral, las Salas de Rehabilitación Integral y los Centros Médicos de Alta Tecnología. Estas unidades aportarían las herramientas para que los médicos cubanos pudieran completar las funciones de la atención primaria de salud, con diagnósticos de certeza y rehabilitación de los pacientes.

Resultado concreto y relevante de este proceso, es la participación masiva de más de 30000 colaboradores de todo el país en sus diferentes perfiles en la Misión Médica Cubana en la República Bolivariana de Venezuela. La provincia Holguín, considerada como una de las abanderadas entre La Habana y Santiago de Cuba por la participación de sus profesionales en esta misión, cuenta con 4161 profesionales y técnicos que han sido colaboradores en ella. Actualmente 2450 colaboradores se encuentran en el cumplimiento de la misión.

\section{La formación de recursos humanos}

Desde el año 1959 el Sistema Nacional de Salud (SNS), ha privilegiado la premisa de que los trabajadores constituyen su principal recurso para lograr su eficacia y su calidad depende del nivel de competencia, actualización de los conocimientos científicos, desarrollo de habilidades técnico profesionales, actitud, valores y cumplimiento de la ética médica de los profesionales que en él laboran (Estado actual de la educación de posgrado de los colaboradores cubanos que cursan especialidades médicas en la Misión Médica Barrio Adentro. Documento de trabajo de la Coordinación Nacional de Docencia. Misión Médica Cubana. 2009).

La formación y el perfeccionamiento de los recursos humanos en los sectores sociales y sanitarios constituyen un componente preciso para el desarrollo sostenible de la sociedad. Y en este caso la formación y capacitación de los recursos humanos para la salud son factores esenciales para garantizar la propia continuidad de la colaboración médica (La educación de posgrado en la Misión Médica Barrio Adentro. Principales resultados y proyecciones. Documento de trabajo de la Coordinación Nacional de Docencia. Misión Médica Cubana. 2009).

Como parte de la estrategia de preparación de los recursos humanos, se han implementado los procesos de formación dentro de las misiones médicas cubanas, esencialmente en la especialidad de medicina general integral. Los colaboradores holguineros han participado activamente en estos procesos en diversos países.

Al ampliarse la cooperación Cuba Venezuela en la formación de los recursos humanos de la salud e intensificarse el trabajo de la Misión Barrio Adentro, Fidel plantea que la misión a desarrollar tenía dos grandes vertientes: ${ }^{5}$

- Garantizar la asistencia médica al pueblo venezolano: "Salvar vidas."

- Atención a la tropa: garantía de condiciones de vida y seguridad y su formación permanente, de ahí que en abril de 2005 expresa:

"Que todos estudien y se preparen. Que todos se preparen para que regresen mejor formados a Cuba." 
El desarrollo de las ideas de Fidel durante estos años han ido concretando que el internacionalismo en salud comprenda no solo una misión humanitaria y político ideológica, sino que conlleve la necesaria complementación con una formación profesional posgradual, según las posibilidades y la calidad requerida, que permita la inserción futura de los médicos cubanos en áreas específicas del desempeño profesional que requiera tanto Cuba, como cualquiera otra nación (La educación de Postgrado en la Misión Médica Barrio Adentro. Principales resultados y proyecciones. Documento de trabajo de la Coordinación Nacional de Docencia. Misión Médica Cubana. 2009).

Durante los 49 años de historia de la colaboración, la formación de recursos humanos es un elemento primordial, unido de manera indisoluble a la asistencia médica.

\section{La educación de posgrado en la Misión Barrio Adentro}

En abril del año 2004, se comienza realmente la formación de posgrado, dando continuidad y culminación de los estudios a residentes provenientes de Cuba, matriculados en las especialidades de Medicina General Integral (MGI) y de Estomatología General Integral (EGI). La provincia Holguín expone un número destacado de profesionales graduados en ambas especialidades (tabla 1). Actualmente, en formación como futuros especialistas, se encuentran a lo largo de todo el país 39 residentes de MGI y 10 de EGI de la provincia.

Tabla 1. Médicos graduados por años como especialistas en MGI y EGI de la provincia Holguín

\begin{tabular}{|l|c|c|c|c|c|c|c|c|c|c|}
\hline Especialidades & 2004 & 2005 & 2006 & 2007 & 2008 & 2009 & 2010 & Total \\
\hline MGI & 298 & 69 & 79 & 32 & 50 & 31 & 7 & 566 \\
\hline EGI & 0 & 0 & 1 & 191 & 10 & 11 & 2 & 215 \\
\hline Total & 298 & 69 & 80 & 223 & 60 & 42 & 9 & 781 \\
\hline
\end{tabular}

Fuente: datos estadísticos Misión Médica Cubana. República Bolivariana de Venezuela.

Como ejemplo de la solidaridad de la revolución cubana, a fines del año 2004 se inició la formación del residente en esta propia especialidad para médicos graduados en Venezuela, contando para el desarrollo del programa, con los escenarios de la Misión Médica Cubana en Barrio Adentro y sus profesores, con la correspondiente asesoría metodológica. De igual manera se desarrolló la formación de odontólogos generales integrales. Se han graduado en estas especialidades un total de 2877 con la entrega de los títulos por las autoridades venezolanas, de ellos 1848 especialistas en MGI y $839 \mathrm{EGI}$.

Durante esta etapa además, se inició la preparación metodológica en el país, del claustro y los profesores que asumirían el papel de facilitadores para el inicio de la formación académica de los 9 perfiles de las diferentes maestrías de amplio acceso con una matrícula de 11082 maestrantes en dos ediciones. La ejecución de las maestrías se desarrolla de forma descentralizada en cada estado, según el programa establecido. La segunda convocatoria contó con una matricula de 237 colaboradores de la provincia.

La tabla 2 refleja los resultados alcanzados en la lera. convocatoria por maestrías de los colaboradores de Holguín. 
Revista Cubana de Salud Pública. 2011;37(3):276-287

Tabla 2. Profesionales graduados en las diferentes maestrías de amplio acceso durante la 1ra. convocatoria de la provincia Holguín

\begin{tabular}{|l|c|}
\hline Maestrías & Graduados \\
\hline Atención integral al niño & 1 \\
\hline Urgencias médicas & 9 \\
\hline Urgencias estomatológicas & 4 \\
\hline Medicina bioenergética y natural & 1 \\
\hline Salud bucal & 2 \\
\hline Longevidad satisfactoria & 2 \\
\hline Enfermedades infecciosas & 4 \\
\hline Total & 23 \\
\hline
\end{tabular}

Fuente: datos estadísticos Misión Médica Cubana. República Bolivariana de Venezuela.

Se han desarrollado maestrías semipresenciales de la Escuela Nacional de Salud Pública de Cuba, las que se han impartido con la participación de los comités académicos y sus claustros de profesores. Los programas se han adecuado en sus estrategias docentes a las condiciones de la República Bolivariana de Venezuela. Han sido dirigidos a la formación de los cuadros docentes y administrativos que se han destacado en su desempeño laboral y como directivos, con lo que se materializa la indicación de Fidel de perfeccionar la preparación de los cuadros para asumir nuevas tareas.

La Maestría en Educación Médica, pilar estratégico en la preparación y formación del profesor y continuidad del diplomado, en su primera edición se inició en noviembre del 2005, con una matrícula inicial de 336 maestrantes. De ellos han defendido sus tesis, en Venezuela y en la Escuela Nacional de Salud Pública, 142 maestrantes (48,3 $\%)$. De la provincia se han graduado 6 maestrantes. Se inició la segunda edición de la maestría en junio de 2008.

Otras como la Maestría en Salud Pública, Farmacoepidemiología y Economía de la Salud, se iniciaron en los años 2007 y 2008 respectivamente. En este grupo de maestrías la provincia Holguín logró graduar cinco colaboradores y tuvo una participación destacada en Farmacoepidemiología y Economía de la Salud con seis profesores.

Con el objetivo de garantizar los recursos humanos para la apertura de los servicios en los Centros Médicos de Diagnóstico Integral (CMDI) en Venezuela, se seleccionaron un grupo de especialistas de MGI para la Misión Haciendo Futuro, que consistió en una preparación intensiva en Cuba, con diplomados en Ultrasonido, Endoscopía, Cuidados Prácticos de Medicina Intensiva, Cirugía, Medicina Física y Rehabilitación (MFR), Oftalmología y Anestesiología. Esta preparación previa a la práctica profesional en los CMDI propició la continuidad de la formación académica de estos profesionales. En el año 2006, comienzan a desarrollarse las especialidades de MFR con una matrícula de 438 residentes e Imaginología con 327 . Actualmente la provincia cuenta con cinco residentes en formación de 2do. año en MFR y graduó a dos profesionales como especialistas en I maginología.

En el año 2007 se inician otras especialidades como Oftalmología con una matrícula de 289 residentes en los escenarios de formación del CMDI, ópticas Populares y

http://scielo.sld.cu 
centros oftalmológicos, Medicina Intensiva y Emergencias con matrícula de 540 residentes, así como Gastroenterología con 287 residentes, el escenario principal de formación en estas especialidades es el CMDI y el Centro Médico de Alta Tecnología (CMAT).

En el año 2008 se comienzan a realizar las especialidades de Anestesiología y Reanimación y Medicina Intensiva y Emergencias con una matrícula de 635 residentes; Cirugía con una matrícula de 47 residentes de primer año y Anestesiología y Reanimación con 14 residentes.

Actualmente la provincia cuenta con profesionales en formación de estas especialidades en los diferentes estados del país (tabla 3 ).

Tabla 3. Total de residentes en formación por especialidad de la provincia Holguín en la Misión Médica de Venezuela

\begin{tabular}{|l|c|}
\hline Especialidades & Residentes en formación \\
\hline Medicina Física y Rehabilitación & 5 \\
\hline Imaginología & 3 \\
\hline Oftalmología & 11 \\
\hline Medicina Intensiva y Emergencias & 14 \\
\hline Gastroenterología & 1 \\
\hline ARMiE* & 8 \\
\hline Cirugía general & 3 \\
\hline Anestesiología y Reanimación & 11 \\
\hline Total & 89 \\
\hline
\end{tabular}

* Anestesiología y Reanimación y Medicina Intensiva y Emergencias Fuente: datos Estadísticos Misión Médica Cubana. República Bolivariana de Venezuela.

El proceso de formación doctoral en Venezuela se inició en el primer semestre del año 2006, identificando 272 médicos, estomatólogos y licenciados, los que iniciaron el trabajo en las diferentes etapas. Se decidió garantizar la continuidad en la patria de todos los colaboradores con requisitos al concluir la misión.

\section{Bases para el desarrollo del pregrado en la República Bolivariana de Venezuela}

El inicio de la Misión Barrio Adentro elevó el número de colaboradores holguineros, de todas las categorías y especialidades, se generó un movimiento importante de profesores y en el 2006 se inicia la formación en Tecnología de la Salud, en la cual se encontraban matriculado 316 holguineros al cierre de diciembre del 2009; nueve de ellos culminaron sus estudios con su examen estatal.

En 2008 se extiende la formación a la Licenciatura en Enfermería, donde ya se encontraban 39 compañeras y compañeros de la provincia. Se cuenta con cuatro 
compañeras que defendieron su examen estatal dentro de la misión graduándose como licenciadasde la especialidad.

En el 2009 se desarrolla el internado profesionalizante en los escenarios de Barrio Adentro, se reciben 500 internos, de ellos 50 son holguineros que se ubican en el estado Distrito capital. Todos realizan un trabajo meritorio y hoy están graduados con la experiencia extraordinaria de la realización de su internado dentro de una misión internacionalista.

\section{El desarrollo del pregrado para los recursos humanos venezolanos en el campo de la medicina. El Programa Nacional de Formación de Medicina Integral Comunitaria}

En octubre del 2005 se da inicio a una colosal tarea en el campo académico, bajo la guía y dirección de Fidel, se trata de la formación de pregrado en Barrio Adentro, tomando en consideración la solicitud formulada por el Gobierno Bolivariano, respecto a la necesidad de formar profesionales de la salud, especificamente médicos, que pudieran dar continuidad a la obra que la Misión Médica Cubana venía construyendo en el país.

Surge así el Programa Nacional de Formación de Medicina Integral Comunitaria (PNFMIC), que tiene la misión de formar en los escenarios de Barrio Adentro en la comunidad a los futuros médicos bolivarianos.

Se enuncia así el perfil del egresado:

El Médico Integral Comunitario es un médico con competencias diagnósticas y terapéuticas, capaz de brindar atención médica integral a través de acciones de promoción, prevención, curación y rehabilitación del individuo, la familia, la comunidad y el medio ambiente; con el empleo de los métodos clínico y epidemiológico, un profundo enfoque social, portador de valores éticos, humanísticos, solidarios y de actitud ciudadana; llamados a transformar la situación de salud en correspondencia con las exigencias de la sociedad actual de la República Bolivariana de Venezuela. ${ }^{5}$

En la hermosa tarea de diseñar la carrera participaron profesores de alto nivel de todo el país y una representación de la provincia, Holguín constituida hasta la fecha por 11 profesores que laboraron en los grupos de diseño curricular nacionales y en el trabajo docente metodológico de los estados.

Por primera vez se hace posible la consigna de que un profesional puede formar a su imagen y semejanza a estudiantes bajo el precepto del trabajo tutorial y la temprana vinculación a la profesión en los escenarios laborales de la comunidad.

Entre los antecedentes por los que se desarrolla el PNFMIC, se puede resumir: ${ }^{3}$

- La práctica médica se basa en un modelo de atención centrado en la enfermedad y lo curativo (Modelo Curativo-Asistencial), de alto consumo tecnomédico, profunda dependencia tecnológica y altos costos, con el subsecuente abandono de los programas preventivos e inexistencia de programas de promoción de la salud.

- Predominio del modelo conceptual biologicista y medicalizado, haciendo que la formación y la investigación se impregnaran del concepto de enfermedad, desvinculándose de la salud y sus determinantes. 
- Una práctica pedagógica circunscrita al aula con unas prácticas profesionales en los últimos años de su carrera, centrada casi exclusivamente en los hospitales.

- Formación de profesionales con escasa sensibilidad social, poca capacidad resolutiva y mayor propensión a la mercantilización y la deshumanización de la atención médica.

- Desarticulación entre la formación de recursos humanos y las necesidades de salud de la población.

Por ello es importante hacer un grupo de reflexiones sobre el modelo de formación del médico propuesto: ${ }^{3}$

- Para poder llevar adelante el nuevo modelo pedagógico, este debe aglutinar en su cuerpo las mejores experiencias de los modelos tradicionales y agregarle la concepción novedosa de la formación desde y dentro del escenario laboral del futuro profesional, la atención primaria de salud.

- El estudiante se inserta desde el inicio de la carrera en un consultorio médico de la atención primaria, lo que permite desde su temprana incorporación desarrollarle los hábitos y habilidades que los prepararán para ejercer su profesión con mayor calidad.

- El profesor del consultorio tiene a su cargo la atención de un grupo determinado de estudiantes, con los cuáles mantiene durante toda la carrera un permanente intercambio, no solo académico, sino también de carácter formativo, que le permite ir modelando los principios de la futura práctica profesional independiente.

- El profesor realiza un trabajo tutoreal permanente, tutor de la adquisición del conocimiento a tutor para la vida, produciéndose una simbiosis que redunda en una elevada preparación científica individual y en un elevado altruismo y espíritu de sacrificio que se crea de forma ascendente en el estudiante.

- La formación del estudiante ocurre en el seno de un núcleo docente, que se organiza a partir de un grupo de consultorios en la comunidad donde vive, y donde realiza su formación, se añade a estos consultorios un aula multipropósito y el conjunto de unidades de salud que garantizan la fortaleza en su preparación científica.

- El proceso de enseñanza-aprendizaje está basado en el aprender a aprender, se organiza de tal manera que se produce una dosificación del contenido, en forma de sistema: pasa de lo más simple a lo más complejo, aprovecha todos los recursos disponibles tanto en medios de enseñanza como en recursos tecnológicos, favorece el autoestudio y una preparación comprometida, que reafirma el compromiso individual del estudiante y fortalece cada vez más el papel orientador del profesor.

- El trabajo grupal en los diferentes momentos del núcleo docente, favorece el desarrollo de la solidaridad, del colectivismo, de los principios éticos, un enfoque de inserción social, la responsabilidad con lo público partiendo de que su aula multipropósito está ubicada y custodiada en el seno de la comunidad. El estudiante se identifica con este espacio con un mayor nivel de pertenencia, y ello da continuidad a la creación de hábitos y conductas sociales de un elevado carácter humanista y de compromiso con su pueblo y su revolución, que viene expresada desde su práctica en el consultorio de atención primaria.

El programa incluye las siguientes modalidades: métodos abiertos y participativos, que tengan como eje la educación en el trabajo en forma semipresencial con 
seguimiento permanente que induzca a la indagación, organización y adquisición de conocimientos, hábitos y habilidades para la resolución de problemas.

Se propone una nueva orientación para la construcción y desarrollo del conocimiento centrándose en la multidisciplinariedad, interdisciplinariedad y transdisciplinariedad, a partir de la estrategia de atención primaria de salud, lo que posibilita nuevas representaciones, complejas y multidimensionales del mundo y del ser humano.

La «Universidad Barrio Adentro» es una concepción y no una edificación, ya que existe y se desarrolla en cada uno de los lugares en que se produce el proceso formativo. No es una universidad que se integra a los servicios de salud, existe como una expresión de mayor desarrollo, fundamentada científicamente, en la función formativa inherente al perfil del desempeño de los medios de Barrio Adentro.

Se estableció como estrategia docente del Programa, la interrelación entre la orientación de la actividad, la consolidación de los conocimientos y habilidades y la evaluación de los resultados del aprendizaje. Conlleva la asignación de tareas que debe cumplir en el proceso docente que se desarrolla en el consultorio (comunidad) y en su trabajo independiente, todo lo que se complementa con el autoestudio.

Y para la garantía del proceso se desarrolla un programa de preparación para todos los profesores que contempla la autopreparación dirigida de los contenidos de ciencias básicas, la preparación básica en educación médica y la preparación técnicometodológica para el desarrollo específico de las diferentes actividades docentes previstas en el Programa.

En la tabla 4 se muestra, al cierre del 2009, el estado de la matrícula.

Tabla 4. Resumen de la matrícula del Programa Nacional de Formación de Medicina Integral Comunitaria al cierre de diciembre de 2009

\begin{tabular}{|l|c|}
\hline Año académico & Matrícula \\
\hline Primero & 4262 \\
\hline Segundo & 4566 \\
\hline Tercero & 5697 \\
\hline Cuarto & 10178 \\
\hline Total & 24703 \\
\hline
\end{tabular}

Fuente: datos estadísticos Misión Médica Cubana. República Bolivariana de Venezuela.

Ya en el año 2010, la carrera se extiende hasta el quinto año.

Como resultados de este proceso, 1187 colaboradores holguineros han participado en el proceso de formación del médico integral comunitario, de ellos 760 especialistas en MGI y 124 obtuvieron la categoría docente de instructor en los procesos desarrollados en la misión.

Como resultado de la necesidad de formación de médicos para Latinoamérica y el mundo, surge a partir del compromiso de Sandino, establecido entre Fidel y Chávez, el Nuevo Programa de Formación de Médicos, que se desenvolvió en cuatro polos del

http://scielo.sld.cu 
país, situados en las provincias Matanzas (Polo Jagüey), Pinar del Río (Polo Sandino), Cienfuegos (Polo Horquita) y la I sla de la Juventud. Este programa se desarrolló con la aplicación de la experiencia obtenida en la República Bolivariana de Venezuela y en él participaron activamente como profesores 269 colaboradores holguineros, especialistas de medicina general integral.

Se materializa la conducción estratégica de la formación de capital humano en el sector de la salud, así como el principio indiscutible de solidaridad de la revolución cubana y la participación de sus trabajadores en el cumplimiento de los objetivos de lograr la excelencia en los servicios de salud y participar activamente en la formación de los recursos humanos para Cuba y el mundo.

\section{REFERENCI AS BI BLI OGRÁFICAS}

1. Marimón Torres N. La colaboración Medica Cubana en el siglo XXI : una propuesta para la sostenibilidad en Guinea Bissau [tesis]. La Habana: Escuela Nacional de Salud Pública; 2006

2. El pensamiento de Fidel Castro Ruz sobre la Educación Médica Cubana. La Habana: Escuela Nacional de Salud Pública; 2005.

3. Borroto Cruz R, Salas Perea R, Díaz Rojas P. Un nuevo modelo formativo de médicos en la Universidad Barrio Adentro, República Bolivariana de Venezuela. Educ Med Super [Internet]. 2010 [citado 9 Oct 2010];24(1):111-35. Disponible en: http://scielo.sld.cu/scielo.php?script=sci_arttext\&pid=S0864$\underline{21412010000100013 \& \operatorname{lng}=\mathrm{es}}$

4. Marimón Torres N, Martínez Cruz E. Evolución de la colaboración médica cubana en 100 años del Ministerio de Salud Pública. Rev Cubana Salud Pública [Internet]. 2010 [citado 9 Oct 2010]; 36(3):254-62. Disponible en:

http://scielo.sld.cu/scielo.php?script=sci_arttext\&pid=S0864 34662010000300010\&lng=es

5. Consejo Nacional de Universidades. Programa Nacional de Formación de Medicina Integral Comunitaria. Caracas. Consejo; 2007.

Recibido: 28 de octubre de 2010.

Aprobado: 9 de noviembre de 2010.

Pedro A Díaz Rojas. Escuela Nacional de Salud Pública. Calle 100 s/n entre Perla y E. Altahabana. Municipio Boyeros. La Habana. Cuba. Correo electrónico:

pdiaz@infomed.sld.cu

http://scielo.sld.cu 